\title{
The Metaphysical Character of the Criticisms Raised Against the Use of Probability for Dealing with Uncertainty in Artificial Intelligence
}

\author{
Carlotta Piscopo • Mauro Birattari
}

Received: 6 February 2007/Accepted: 21 April 2008/Published online: 16 May 2008

(C) Springer Science+Business Media B.V. 2008

\begin{abstract}
In artificial intelligence (AI), a number of criticisms were raised against the use of probability for dealing with uncertainty. All these criticisms, except what in this article we call the non-adequacy claim, have been eventually confuted. The non-adequacy claim is an exception because, unlike the other criticisms, it is exquisitely philosophical and, possibly for this reason, it was not discussed in the technical literature. A lack of clarity and understanding of this claim had a major impact on AI. Indeed, mostly leaning on this claim, some scientists developed an alternative research direction and, as a result, the AI community split in two schools: a probabilistic and an alternative one. In this article, we argue that the nonadequacy claim has a strongly metaphysical character and, as such, should not be accepted as a conclusive argument against the adequacy of probability.
\end{abstract}

Keywords Artificial intelligence - Probability · Alternative approaches · Randomness · Uncertainty

\section{Introduction}

Antony Eagle (2005) has recently argued that the concept of randomness, as presented in the current philosophical literature, is misleading and that this can entail serious consequences due to the central role that randomness plays in many scientific disciplines.

C. Piscopo $(\bowtie) \cdot$ M. Birattari

IRIDIA, CoDE, Université Libre de Bruxelles, Brussels, Belgium

e-mail: piscopo@iridia.ulb.ac.be

M. Birattari

e-mail: mbiro@ulb.ac.be 
In this article, we discuss the implications that the lack of an adequate definition of randomness had in the development of artificial intelligence. From the late 1960s till the late 1980s, the artificial intelligence community engaged in a vigorous and at times vitriolic debate about the nature of uncertainty and about the methods for its treatment. A part of the community considered probability as perfectly adequate for dealing with uncertainty. Another part of the community insisted instead on the inadequacy of the probabilistic framework and devoted itself to the development of alternative approaches including, for example, fuzzy sets theory (Zadeh 1965), possibility theory (Zadeh 1978; Dubois and Prade 1987), Dempster-Shafer theory (Shafer 1976), and the transferable belief model (Smets and Kennes 1994). For a broad overview on the debate see Shafer and Pearl (1990). As a result of this debate, the artificial intelligence (AI) community is nowadays split in two schools that develop along distinct research directions. This appears clearly from the fact that a significant amount of research works dealing with alternative methods are published in journals or conference proceedings that are entirely devoted to them. The fact that the debate on uncertainty in AI reached its climax about 20 years ago, and that since then only few articles have been devoted to this debate, does not mean that a satisfactory definition of uncertainty, and of the related concept of randomness, has been achieved. Indeed, the very existence of the two communities should be seen as an evidence of the fact that some key issue on which these two communities diverge has not been properly addressed. Our thesis is that the key issue is what we call here the non-adequacy claim.

All the criticisms raised against the adoption of probability in AI have been discussed at length in the literature and have been eventually confuted, typically by highlighting some technical fault. A systematic analysis of the discussion of these criticisms can be found in Cheeseman (1985). Possibly, the only criticism that has not been addressed is the non-adequacy claim, which concerns the status of randomness that is assumed within the probabilistic framework. This claim has been proposed in different formulations and can be summarized as follows:

Probability theory is not suitable to handle uncertainty in AI because it has been developed to deal with intrinsically stochastic phenomena, while in AI uncertainty has an epistemic nature.

Contrary to most of the other criticisms moved against the use of probability in AI, the non-adequacy claim has a marked philosophical character. Plausibly for this reason, the non-adequacy claim does not lend itself to the kind of analysis that is customarily proposed in the technical literature. As a consequence, this claim has been so far overlooked.

The separation of the AI community in two schools is now perceived as highly problematic: In their first editorial on Fuzzy Sets and Systems, the most representative journal of the alternative school, the current editors stress that establishing a common ground with the mainstream is one of their priorities (Dubois and Prade 1999). This appears unfeasible unless the non-adequacy claim is properly addressed. By taking a philosophical outlook on the non-adequacy claim, we show that it has a strongly metaphysical character and that, as such, it should not be accepted as a conclusive argument against the adequacy of probability. 


\section{The Critical Issue of Uncertainty in AI}

Uncertainty is a key issue in AI. Indeed, the final goal of AI is to design artifacts that are able to carry out tasks in the real world without human intervention. These artifacts should gather information on the environment and, on the basis of this information, make decisions and act purposely. In the typical case, the knowledge gathered on the environment is incomplete and/or imprecise, which calls for the adoption of decision making procedures that are robust to uncertainty.

As detailed in Section "The Emergence of Uncertainty", issues related to uncertainty characterized only an advanced phase of the development of AI. At that moment, probability appeared as the natural candidate for dealing with uncertainty. Yet, as we will see in Section "Criticisms against Probability, Answers and Open Questions", criticisms were raised on the adequacy of the probabilistic approach.

\section{The Emergence of Uncertainty}

In its early developments, AI did not have to handle the problem of uncertainty. The early machines were designed to perform intellectual activities like proving theorems (Newell et al. 1957) or playing chess (Newell et al. 1958). In these applications, the world experienced by machines is an abstract world of which a complete model is provided in the form of a symbolic description. In the case of chess, for example, a full description of the environment can be given by specifying the position of each piece on the chessboard and the immediate result of each move is fully predictable. Chess is a game with perfect information since the current state of the game is known at any time by both players, moreover, chance does not play any role since the rules of the game are rigidly deterministic. It was precisely the noise-free character of this game that made it the perfect application to try out the newborn AI techniques. Early AI researchers were confident that the high speed of computers could overcome the main issue in chess playing, that is, the huge size of the state space. Indeed, the typical chess program is designed to explore in a more or less exhaustive way all possible moves and counter-moves down to a certain depth, for then selecting the most promising move on the basis of some criterion. Yet, this brute-force approach is not viable in real-world contexts: Besides the speed needed for evaluating a possibly large number of alternative options, real-world applications require the ability to take decision and act on the basis of typically partial and/ or imprecise knowledge.

Artificial intelligence techniques started being applied to real-world problems between the late 1970s and the early 1980s. Nonetheless, in these first real-world applications, the problem of uncertainty was substantially dodged. In mobile robotics, for example, robots were navigating in toy worlds rather than in the real one: the environment they were moving in was highly engineered and the operating conditions were carefully controlled. The robotics project Shakey (Nilsson 1984) is a significant example of this phase of the research in mobile robotics and more generally in AI. Shakey's world is designed to resemble as close as possible a chessboard, where objects are assigned a well defined location and are easily 
detectable. This clearly simplifies both the perception and the representation of the environment. ${ }^{1}$ Like in the case of chess, the model of the environment Shakey is equipped with, is symbolic and it is supposed to be a perfect mirror of the (simplified) world in which Shakey navigates. The Shakey project apparently rests upon the assumption that techniques designed for operating a robot in a simplified world would directly generalize to the real world. In the late 1970s and early 1980s, the same assumption underlined the newborn expert systems designed to help human experts to make decisions within a specialized area of knowledge. The world to which expert systems (Shortliffe 1980; Buchanan and Shortliffe 1984; Buchanan and Feigenbaum 1978; Duda et al. 1979) refer is a simplified and self-contained version of the real one.

The late 1980s and early 1990s saw the decline of the symbolic approach. With a provocative article titled "Elephants don't play chess", Rodney Brooks opened the way to what he called the nouvelle AI (Brooks 1990). He questioned the grounding idea of the abstract artificial intelligence (Brooks 1990, 1991) according to which intelligence consists in performing intellectual activities in an abstract world like that of chess. In what can be considered a manifesto of the nouvelle AI (Brooks 1991) Brooks stated that the time had come to focus on embodied systems that are situated in real worlds scenarios and that are able to react to contingencies on a realtime basis. As Brooks pointed out (1991) between the 1980s and the 1990s, almost all researchers had access to fast and powerful processors: huge amount of realworld data, and the uncertainty connected to it, could be processed in a reasonable amount of time and at a reasonable cost. Besides, starting from the 1980s, huge investments were devoted to the research aiming at building systems capable of moving purposefully in real-world scenarios without human intervention. ${ }^{2}$ The growing number of implementations of physical systems situated in the real world made clear that real-world scenarios should be considered in all their complexity and that the uncertainty associated with these scenarios should not be dodged but, rather, properly handled.

\section{Criticisms Against Probability, Answers and Open Questions}

When AI applications had to explicitly deal with uncertainty, the probabilistic framework appeared as the natural candidate for building models from partial knowledge and for supporting decision making under uncertainty. The probabilistic framework was a well-established 300-year-old framework with a respectable record of successes in treating practical problems emerging in many scientific domains ranging from statistical mechanics to information theory. Nevertheless,

\footnotetext{
1 The chessboard model of the environment has been adopted in several AI applications. In machine learning, for example, different reinforcement learning (Sutton and Barto 1998) techniques such as Qlearning (Watkins 1989) are designed to tackle problems in which the world is represented as a grid and in which objects are represented as points inhabiting the sectors of this grid.

2 Just to cite one significant example, the Defense Advanced Research Projects Agency-the agency of the US Department of Defense that is responsible for the development of new technologies for military use-sponsored a robotics research project with the goal of developing an autonomous vehicle running on roads.
} 
following McCarthy and Hayes (1969), some researchers criticized the use of probability in AI applications and, accordingly, they developed alternative formalisms.

The analysis we propose in this article stems from the understanding that the criticisms raised against the probabilistic framework are indeed composite. We find convenient to highlight two main elements in these criticisms. The first one is related to technical issues on the use of probability theory, while the second one is clearly of philosophical nature and concerns the status of the hypotheses assumed within such a framework.

The technical element was discussed at length in the artificial intelligence literature: both the arguments against probability theory and the counter-arguments were clearly expressed and analyzed. A systematic and authoritative analysis was proposed in the mid-1980s by Peter Cheeseman (1985). The fundamental idea underlying Cheeseman's counter-arguments (1985) is that most criticisms raised against probability theory derive from a unique fallacy, namely the idea that probabilities have to be conceived as frequency ratios. Indeed, according to Cheeseman, most criticisms state that probability is not appropriate for dealing with those situations in which repeated experiments cannot be performed and where, thus, the frequency ratio definition is meaningless. Cheeseman pointed out that a Bayesian interpretation is all what is needed when coping with little data, which is the usual case in AI applications.

Cheeseman's stand for a Bayesian subjective interpretation of probability in AI is indeed the cornerstone of his defense of probability. A number of other elements in his analysis followed naturally.

According to Cheeseman, the recurrent statement "Bayesian analysis requires vast amounts of data" (Buchanan and Shortliffe 1984) and the strictly related "prior probabilities assume more information than given" (Garvey et al. 1981; Buchanan and Shortliffe 1984) derive from the above mentioned fallacy. Cheeseman pointed out that when knowledge is lacking, the maximum entropy assumption allows one to make the initial assignments. If the updated assignments differ from the initial ones, we do not have to conclude that the initial ones were wrong. Such conclusion would be again based on a misconception, namely that we deal with the true probability of an hypothesis. From his subjective Bayesian position, Cheeseman made clear that probability assignments are simply numerical values that express our state of knowledge and that can evolve, even significantly, due to the fact that we acquire further information.

Another claim analyzed by Cheeseman is that "more than one number is needed to represent uncertainty" (Shafer 1976). Cheeseman elaborated on the issue pointing out that different representations can be considered. A selection among them should be based on their capability of successfully solving the specific problem at hand.

Cheeseman addressed also the widely heard statement that the Bayesian approach is not appropriate since "people are not good in estimating probabilities" (Tversky and Kahneman 1974). This incapability of estimating probabilities emerged clearly in the domain of expert systems, where human experts turned out to be often unable to provide consistent prior probabilities. According to Cheeseman, we should not conclude that probability is inadequate, but simply tackle this problem. Also in this 
context, Cheeseman pointed out that when knowledge is not enough for defining prior probabilities, the maximum entropy should be used.

With his article, Cheeseman thought he had provided conclusive counterarguments to all claims raised against the use of probability in AI. The same conviction was shared by a vast majority of the AI community, which felt that the debate on the adequacy of probability could be considered as fully extinguished. Nonetheless, it was recognized that the debate had been fruitful and raised some genuine issues that stimulated further research. ${ }^{3}$ For example, Bayesian networks (Pearl 1988) emerged as a result of the discussion. The significant successes obtained by Bayesian networks in the solution of problems involving uncertain knowledge strengthened further the conviction that probability is all what is needed when dealing with uncertainty.

On the other hand, some researchers kept feeling unsatisfied with the probabilistic framework and kept pursuing the development of alternative approaches. In Section “The Metaphysical Character of the Non-adequacy Claim”, we argue that the lack of a thorough analysis of the philosophical element, embodied by the non-adequacy claim, is what determined the separation between the two schools.

\section{The Metaphysical Character of the Non-adequacy Claim}

The fact that the mainstream keeps using the probabilistic framework indicates that no conclusive empirical evidence has been produced in the literature showing that handling AI problems in probabilistic terms systematically leads to incorrect predictions. This suggests that the justification of alternative approaches stands on non-evidential arguments. Our thesis is that the argument that justified the introduction of alternative methods, and determined the split of the AI community, is the non-adequacy claim. Our analysis will show that this argument has a marked metaphysical character in Popper's sense (Popper 1935), that is, this argument cannot be tested and possibly disproved on the basis of experience.

In Section "Two Formulations of the Non-adequacy Claim", we discuss two different forms under which the non-adequacy claim appeared in the AI literature. In Section "Challenging the Non-adequacy Claim", we point out why this claim entails a metaphysical drift.

Two Formulations of the Non-adequacy Claim

The non-adequacy claim recurs particularly often in the technical literature. In this section, we discuss two versions of the non-adequacy claim that can be found in the writings of two of the most representative scientists of the alternative school: Lotfi Zadeh and Glenn Shafer. Yet, before entering the discussion of these two versions, we briefly introduce their general characterizing elements.

\footnotetext{
3 For a broad analysis on the advancements made in the understanding and in the treatment of uncertainty in the last 20 years see (Walley 1991, 1996, 2000).
} 
Both versions stand upon a dichotomy. Zadeh and Shafer assume that uncertainty has two distinct natures: a stochastic and an epistemic one. A dichotomic view of uncertainty is not new. Indeed, for centuries uncertainty has been interpreted by some authors as stochastic and by some others as epistemic. See Hacking (1975) for a remarkable historical analysis of this duality. Concerning the dual nature of uncertainty, the point made by Zadeh and Shafer contains an element of novelty. They state that both a stochastic and an epistemic uncertainty exist. They argue that these two kinds of uncertainty are distinct and of different nature. They conclude that the two kinds of uncertainty need to be handled with two distinct approaches. Zadeh and Shafer consider probability to be adequate to handle stochastic uncertainty, while they propose fuzzy sets theory and Dempster-Shafer theory, respectively, to handle epistemic uncertainty. According to them, whether one should adopt a probabilistic model or an alternative one in order to tackle a given problem depends uniquely on the nature of the problem at hand: A problem is stochastic if the source of uncertainty can be traced back to an underlying physical random mechanism; conversely, a problem is epistemic, and therefore deterministic, if no underlying random mechanism can be spotted. In the latter case, alternative methods are adequate since no randomness hypothesis is postulated in the mathematical apparatus. The adoption of two types of models ensures that every kind of uncertainty is handled by a framework that is specifically tailored to it, that is, that matches its inherent nature.

In this argument, we find convenient to isolate two main hypotheses. We name them discrimination hypothesis and correspondence hypothesis. On the one hand, the discrimination hypothesis assumes that it is possible to draw a sharp demarcation line between what is inherently stochastic and what is inherently deterministic. On the other hand, the correspondence hypothesis assumes that, in order to be adequate, a model has to match the inherent nature of the given problem. In Section "Challenging the Non-adequacy Claim", we will critically analyze these two hypotheses and we will show that they raise a number of issues. Here, we limit ourselves to use the above concepts to disentangle the main passages of Zadeh's and Shafer's argumentation.

\section{The Non-adequacy Claim in Zadeh}

The following passage of Zadeh constitutes an illustrative example of the kind of dichotomic argumentation we sketched above:

In dealing with soft data of the type encountered in such diverse fields as psychology, sociology, anthropology, medicine, economics, management science, operation research, pattern classification and system analysis, it is a standard practice to rely almost entirely on the techniques provided by probability theory and statistics [...]. It can be argued, however, as we do in the present article, that such techniques cannot cope effectively with those problems in which the softness of data is nonstatistical in nature-in the sense that it relates, in the main, to the presence of fuzzy sets rather than to random measurement errors or data variability-(Zadeh 1981 pp. 69-70). 
On a strictly technical level, this statement of Zadeh has been already discussed by Cheeseman (1985) who refuted it by showing that the founding concepts of fuzzy sets theory-such as the concepts of fuzzy sets and degree of membership (Zadeh 1965) — can be formulated in purely Bayesian terms.

As far as our analysis is concerned, in this quotation we can recognize the discrimination hypothesis behind the distinction that Zadeh draws between problems that are "nonstatistical in nature" and those that are not so; and the correspondence hypothesis behind the claim that the former cannot be modeled in probabilistic terms since no random process is involved. Zadeh instantiates the terms of the above distinction by the dichotomy randomness/imprecision. In particular, he stresses the idea that the imprecision of natural language is not to be assimilated to randomness since it has an intrinsically deterministic nature. In his view, imprecision is deterministic because:

the source of imprecision is the absence of sharply defined criteria of class membership, rather than the presence of random variables-(Zadeh 1965, p. 339).

The source of imprecision is simply ascribed to the fact that the problem at hand is not sharply defined. What Zadeh means by this is made clear by the wording "presence of random variable". By adopting the term presence, Zadeh conveys the idea that a random variable is not a mathematical entity but, rather, an entity that exists in Nature. If this entity can be spotted in the portion of reality we are observing, it is legitimate to adopt a probabilistic framework. Otherwise, it means that we are faced to a deterministic, but not well defined, problem and fuzzy sets have to be employed.

An example can better clarify Zadeh's viewpoint. In the statement "John is tall", uncertainty comes from the fact that tallness is not sharply defined and is rather a gradual property (Bellman and Zadeh 1970, p. 142). Zadeh handles the problem by reformulating the original statement as "John is a member of the class of tall people", and by introducing the concept of degree of membership: the higher the degree of membership of John into the class of tall people, the taller John is. On the contrary, in Zadeh's terminology, the statement "John will get married within a year" is "a probabilistic statement concerning the uncertainty of the occurrence of a nonfuzzy event" (Bellman and Zadeh 1970, p. 142). Here, uncertainty stems solely from the fact that it is not known precisely when the sharp event "marriage" will occur. In this case, probability is the right apparatus.

Within this reasoning, the demarcation between deterministic problems and stochastic problems is presented as something objective, existing independently from the observer and the observation. As a consequence, the selection of the model to handle the problem at hand appears to be as a straightforward procedure that simply consists in picking out the model that reflects the nature of the problem itself. This emerges clearly in Zadeh's claim that fuzzy sets are the "natural way" (Zadeh 1965 , p. 339) for dealing with epistemic uncertainty and this because they are "completely nonstatistical in nature" (Zadeh 1965, p. 340) as the problem they are intended to model. Here, no empirical evidence is advanced to support the claim: The justification of fuzzy sets stands entirely on the a priori assumptions that we can 
always draw a sharp line of demarcation between different natures of uncertainty and consequently we can select the model that perfectly matches the case at hand.

The discrimination and the correspondence hypotheses have been adopted by Zadeh for justifying also a later development of fuzzy sets theory, that is, the theory of possibility (Zadeh 1978). This kind of justification persists also in his recent scientific works (Zadeh 2005) and clearly appears to have influenced the literature dealing with alternative methods. Just to cite a representative example, a justification of the fuzzy approach based on the discrimination and the correspondence hypotheses can be found substantially unaltered in a very recent work by Ashu Solo and Madan Gupta (2007 p. 251). ${ }^{4}$

\section{The Non-adequacy Claim in Shafer}

In its main traits, Shafer's argument resembles the one proposed by Zadeh. Also Shafer's version of the non-adequacy claim stands on a dichotomy: the dichotomy chance/belief. Shafer introduces this dichotomy through an analysis of its historical evolution and argues that, for centuries, the concept of belief has been identified with that of chance and that these two concepts have been wrongly unified under the name of probability (Shafer 1976, 1978). Since the dawn of probability, epistemic uncertainty and aleatory uncertainty have been clearly distinguished, being the former the older one. Yet, with the introduction of the theory of chances in the late seventeenth century "probability acquired its aleatory connotations" (Shafer 1976 p. 33) and the two kinds of uncertainty have been superposed. Shafer argues that this superposition is misleading, and that, while chance is an inherent property of a random experiment, belief is a personal opinion about the outcome of such an experiment. Since they do not necessarily coincide, it is a forcing fit to make beliefs "obey to all rules obeyed by chance" (Shafer 1976, p. 9). The demarcation should be restored and the two kinds of uncertainty should be handled by two different techniques. Only chance should obey the axioms of probability, while beliefs should be dealt with alternative rules.

As we can see, the argument that chance and belief have to be treated by different models, is justified on the demarcation and the correspondence hypothesis. Like Zadeh, Shafer assumes that a sharp distinction between epistemic uncertainty and aleatory uncertainty can be done, which allows one to select the model that fits the problem at hand. The fact that the problem is inherently random constitutes the necessary and the sufficient condition for applying probability. If this is not the case, alternative frameworks have to be chosen instead. This idea comes out very clearly in his claim that "chances arise only when one describes an aleatory (or random) experiment" (Shafer, 1976, p. 9). By using the verb arise Shafer appears to meet Zadeh's view according to which chances are not mathematical entities for

\footnotetext{
4 This article appears in a collection of works that report the cutting-edge research in fuzzy theory and soft computing. Within this context, the authors draw a demarcation between type one uncertainty and type two uncertainty, meaning by this stochastic uncertainty and deterministic uncertainty, respectively. Then, on the basis of a correspondence-like argumentation, they claim that "fuzzy logic has proven to be a very promising tool for dealing with type two of uncertainty" and they conclude: "Stochastic theory is only effective with type one uncertainty" (Solo and Gupta 2007, p. 257).
} 
modeling phenomena, but rather real entities that only probability can mirror since it is rooted into the concept of chance.

Yet, unlike Zadeh, Shafer avoids the misconception that Cheeseman (1985) singled out in most of the criticisms raised against probability (see Section "Criticisms against Probability, Answers and Open Questions"), namely that probabilities are necessarily frequencies. Indeed, Shafer interprets probabilities in the Bayesian sense. Nevertheless, he maintains that Bayesian probability is inadequate for dealing with beliefs precisely because beliefs have nothing to do with the chance inhabiting the world, and this is why they have to be ruled by different laws.

More technically, Shafer criticizes the use of Kolmogorov's probability axioms in the definition of a theory of beliefs. In particular, he questions the additivity axiom. He criticizes the fact that this axiom entails that a belief on a hypothesis should be functionally related with the belief on its negation. Coherently, he proposes a new model for dealing with beliefs. This model, named Dempster-Shafer model (Shafer 1976), is an extension of the model developed in the 1960s by Arthur Dempster (1967) but completely departs from the latter precisely in the fact that it does not postulate the third axiom of probability. As we reported in Section "Criticisms against Probability, Answers and Open Questions", the technical questioning of the additivity axiom embodies an actual difficulty encountered by the AI researchers in representing partial beliefs. It is indeed a fact that in some AI applications, like expert systems, practitioners found unnatural to represent partial knowledge in terms of precise additive probabilities. Yet, as already remarked by Cheeseman (1985) (see Section "Criticisms against Probability, Answers and Open Questions"), the rejection of the probabilistic framework should be based on cogent pragmatic reasons. As it appears from the above analysis, Shafer's justification of an alternative framework is, on the contrary, independent from any practical evaluation.

Shafer does not provide any conclusive empirical evidence showing that modeling beliefs in probabilistic terms leads to contradictions. Instead, he grounds his rejection of the probabilistic approach on unempirical conjectures as the one that beliefs cannot be modeled as if they were chances since:

Chances [...] must be conceived as features of the world. They are not necessarily features of our knowledge or belief-(Shafer 1976 p. 16).

Shafer's statement that Nature is inherently stochastic concerns issues that cannot be tested directly. Yet, Shafer insists that handling beliefs as if they were chances is a contradiction in terms. Following this line of reasoning, he criticizes Laplace on his use of probability by saying that:

as a determinist he could not make philosophical sense of randomness(Shafer 1976, p. 17).

The contradiction Shafer sees derives only from the assumption that a model is justified uniquely by its perfect correspondence to the nature of the object it refers to. Coherently with this assumption, adopting a probabilistic framework amounts to assume that randomness is an entity existing in Nature, which clearly could not be 
accepted by a determinist like Laplace. Here, it appears that the reason why Shafer, as well as Zadeh, claims that probability is not adequate to treat epistemic uncertainty has to be searched in the interpretation of the status of randomness. A thorough analysis of this issue is the subject of Section "Challenging the Nonadequacy Claim".

\section{Challenging the Non-adequacy Claim}

The two versions of the non-adequacy claim discussed in Section "Two Formulations of the Non-adequacy Claim" state that it is contradictory to apply the probabilistic framework to problems that are not inherently stochastic. According to this claim, probabilistic models are adequate only when the source of uncertainty can be traced back to some underlying physical stochastic process. If this is not the case, alternative models have to be chosen instead. In this section, we challenge this claim. More precisely, we show that the demarcation and the correspondence hypotheses have a marked metaphysical character and, as a result, we question the non-adequacy claim as a valid argument against the probabilistic framework.

Within the demarcation hypothesis, Zadeh and Shafer adopt a naturalistic interpretation of randomness. In their argumentation, the demarcation between stochastic and deterministic phenomena is presented as something that is in the nature of things: whether a phenomenon is stochastic or deterministic can be simply assessed by direct observation. In reality, such a natural demarcation cannot be drawn on the basis of empirical data. Indeed, empirical data is always finite and, on the basis of a finite sequence of observations, it is not possible to state in absolute terms whether an observed mechanism is stochastic or deterministic. At most, a conventional demarcation can be defined. In the literature, different conventional criteria have been proposed including a characterization of randomness in terms of complexity of the sequence of observations (Kolmogorov 1963) and an operational definition of randomness based on a number of empirical tests (Martin-Löf 1966; Marsaglia 1995). It is in the nature of these criteria to provide conclusions that cannot be taken as absolutely certain.

The above consideration about the distinction between deterministic and random phenomena, highlights an evident clash within the non-adequacy claim. More precisely, it highlights the metaphysical character of the demarcation hypothesis. The fact that many processes exist that, at least up to a certain level of accuracy, can be conveniently described in terms of stochastic variables is justified on the fact that this idealization allows satisfactory results to be obtained when solving problems of interest in several scientific disciplines. As pointed out above, it is yet a completely different issue to establish empirically whether processes exist that are stochastic, that is, to establish if stochastic variables exist in Nature. Indeed, wondering about the actual existence in Nature of stochastic variables is a purely metaphysical issue since it is impossible to falsify, on the basis of a limited number of empirical observations, either the statement "stochastic variables exist in Nature" or its contrary "stochastic variables do not exist in Nature". A stochastic variable is 
simply an entity that is perceived through some observables which take on, in time, values that are governed by some probability measure. In this sense, a stochastic variable is the objectification of the concept itself of a probability measure. Being this concept well defined from a formal viewpoint (Billingsley 1986), stochastic variables exist as mathematical entities in the same way as, for example, real numbers exist.

As far as the correspondence hypothesis is concerned, it is to be observed that this hypothesis contradicts the scientific praxis. Indeed, the adoption of probability in clearly non-stochastic contexts is a very old practice. This idea can be found already in Pascal's argument du pari. Pascal makes precisely the assumption that the very existence of God is the result of a stochastic experiment. Within the Pascalian epistemology, this assumption is clearly only a convenient hypothesis. In the historical development of probability theory, what remained of the pari is the idea that games of chance can be considered as models of all the situations involving uncertainty (Hacking 1975), even for those in which no stochastic experiment is involved. By his hypothesis, Pascal opened the way to the applicability of the probabilistic framework to situations in which little or no data at all is available. Pascal could make use of the probabilistic framework, even if he embraced a deterministic metaphysics, precisely because he intended randomness as an idealization rather than as a noumenon, which appear yet superposed within the non-adequacy claim. It is precisely this superposition that can explain why Shafer (1976) criticizes those determinist scientists, like Laplace, who followed Pascal's idea. As Shafer (1976) correctly remarks, a determinist like Laplace could not accept the idea that Nature is intrinsically stochastic. In fact, intended as an ontological truth, randomness would have clashed unacceptably with the rigid determinism that characterized the philosophy of Laplace. Yet, Laplace accepted randomness simply because he intended it as a convenient fictitious hypothesis for solving practical problems. If randomness is interpreted in this sense, the conflict that Shafer (1976) spots in Laplace's thinking simply disappears.

For centuries after Pascal and Laplace, the probabilistic framework continued to be used in clearly non-stochastic contexts. A significant example is given by nonlinear deterministic systems that present a chaotic behavior. ${ }^{5}$ Despite their deterministic nature, these systems are described and studied with tools such as Markov models and Monte Carlo methods, which were originally developed for stochastic processes. As an example, consider (pseudo-)random number generators commonly used in computer programming. Although these generators are perfectly deterministic, the sequences of numbers they produce pass a variety of tests of randomness and the fact that they look as random is indeed their raison d'etre. If one had to adopt the correspondence hypothesis, these sequences of numbers, being intrinsically deterministic, could not be described in probabilistic terms and could not therefore serve their purpose of being used in computer programs as a source of randomness.

\footnotetext{
${ }^{5}$ It is worth pointing out here that the issue we have raised above against the demarcation hypothesis is not relevant in this context. Indeed, we are concerned here with a mathematical system: the fact that it is deterministic can be stated on the basis of a formal analysis and does not need to be assessed empirically.
} 
In some sense, the correspondence hypothesis is an a priori criterion for assessing the adequacy of a model: It is only the inherent nature of the problem at hand that decides whether a model is adequate or not. This clearly contrasts with the widely accepted scientific practice that prescribes that models should be empirically tested and therefore accepted on the basis of an a posteriori criterion. The shift of the focus from an a posteriori criterion for the assessment of a model to an a priori one, definitely characterizes the correspondence hypothesis as metaphysical.

The metaphysical glow that pervades the whole non-adequacy claim is, in our view, what prevented Zadeh and Shafer from recognizing that the adoption of probabilistic models to deal with epistemic uncertainty stands simply on a working hypothesis: observations of a deterministic but (partially) unknown phenomenon can be seen as if they were produced stochastically. Though fictitious, this hypothesis finds its justification in the fact that it allows one to formulate predictions that are eventually empirically tested. Whether this working hypothesis should be adopted or rejected, ultimately depends on its usefulness in the specific application at hand and can be decided only on the basis of an empirical evaluation. In this sense, the claim that continues being put forward in the recent literature (Zadeh 2005; Solo and Gupta 2007) that probability is inadequate for dealing with the uncertainty arising in deterministic contexts, cannot be accepted as a valid argument against probability.

Keeping to overlook the metaphysical character of this claim and accepting it as such is not without consequence. First of all, this means to preserve the reason of the split between the probabilistic and the alternative school. Indeed, since a metaphysical statement cannot, by definition, be empirically checked, it is accepted or rejected dogmatically. No agreement can be found on such an absolute ground. Until the focus is redirected toward cogent empirical evaluations that the whole community can examine, there will always be an insurmountable reason of separation between the two schools. Secondly, failing to recognize the metaphysical character of the non-adequacy claim could have an impact on the foundations of AI, more in general. If metaphysical criteria like the perfect adherence to the nature of problems were accepted, this would open the way to the rejection, on the basis of metaphysical arguments, of any scientific hypothesis formulated in AI. This because the scientific method allows to put forward hypotheses that are checked against experience but that are never definitively verified, though these are firmly fastened to reality by their ability to solve practical problems.

It is worth pointing out here that the selection among empirical models, that solve equally well a given problem, can be regulated also by extra-evidential criteria on the basis of some principle of economy as for instance simplicity. It should be remarked, for what concerns this issue, that some exponents of the alternative school claimed that the methods they proposed had to be preferred to probability because their analytical formulation turned out to be easier to deal with (Bellman and Zadeh 1970; Dubois and Prade 1997). Yet, this claim appears to be purely qualitative since no systematic study exists, as far as we know, that provides quantitative elements that support the claim. Indeed, no formal ergonomic evaluation has been performed in order to show that alternative methods are simpler. 
Before concluding, we want to add a clarification about our argumentation concerning the use of metaphysical statements in science. By pointing out the metaphysical character of the non-adequacy claim, we do not mean to exclude it tout court. Although we deem it an invalid argument against the use of probability, we do not exclude that this claim could have a regulative role. In other words, we do not exclude that such a metaphysical idea could suggest new techniques for handling uncertainty. After all, in the history of science bold speculations have always played an important role and some of them, thanks to the perseverance of brilliant and far-seeing minds, eventually became scientific theories. This, yet, could happen because these bold speculations withstood the test of experience. The point is that metaphysical ideas can suggest interesting research lines along which new techniques can be conceived, but these techniques will have to be evaluated for their ability to produce satisfactory predictions: Metaphysical elements should not play any role in their evaluation.

\section{Conclusion}

The tangible effect of the lack of a philosophical analysis of the non-adequacy claim is that part of the AI community is nowadays convinced that the introduction of alternative method represents a paradigm shift (Blair1999; Nikravesh 2007). Yet, a paradigm shift requires that an insolvable anomaly is spotted in the current paradigm. In the case of the probabilistic framework, no unsolvable anomaly has been actually highlighted. This explains why the mainstream AI community kept working within the probabilistic approach.

The problem is that nowadays an alternative framework exists, to which a considerable amount of theoretical and experimental work has been devoted. The situation is rather atypical: a 40-year-old framework, which is considered by some as a new paradigm, co-exists with a well-founded 300-year-old one. Notwithstanding many efforts to reconcile these two frameworks, they keep developing along distinct research directions.

In this article, we argued that this situation should be ascribed to a metaphysical quarrel and, as such, it could be solved only by taking a philosophical perspective. A philosophical reassessment of the debate over uncertainty appears to be important for AI. Given the key role that the treatment of uncertainty has in artificial intelligence, an answer to the philosophical questions connected with it could have a significant impact not only on the methodological foundations of the uncertainty field but also on the foundations of the whole AI research. Indeed, inquires about the issue of assessing whether an empirical framework should be accepted or rejected are essential to the foundations of any science and this is even more true for a young discipline like AI that is still in deep evolution.

Acknowledgments Carlotta Piscopo acknowledges the support of a Training Site fellowship funded by the Improving Human Potential (IHP) programme of the Commission of the European Community, Grant HPMT-CT-2000-00032. Mauro Birattari acknowledges support from the fund for scientific research F.R.S.-FNRS of Belgium's French Community, of which he is a Research Associate. 


\section{References}

Bellman, R. E., \& Zadeh, L. (1970). Decision making in a fuzzy environment. Management Science, 17(4), 141-164.

Billingsley, P. (1986). Probability and measure (2nd ed.). New York, NY, USA: Wiley.

Blair, B. (1999). Famous people: Then and now. Lotfi Zadeh. Creator of fuzzy sets. Azerbaijan International, December. Interview with Lotfi Zadeh.

Brooks, R. (1990). Elephants don't play chess. Robotics and Autonomous Systems, 6, 3-15.

Brooks, R. A. (1991). Intelligence without reason. In Proceedings of the International Joint Conference on Artificial Intelligence, pp. 569-595, Morgan Kaufmann Publisher, San Mateo, California.

Buchanan, B., \& Feigenbaum, E. (1978). Dendral and meta-dendral: Their applications dimension. Artificial Intelligence, 11(1, 2), 5-24.

Buchanan, B. G., \& Shortliffe, E. H. (Eds.). (1984). Rule-based expert systems. The MYCIN Experiments of the Stanford Heuristic Programming Project. Reading, MA, USA: Addison-Wesley.

Cheeseman, P. (1985). In defense of probability. In Proceedings of the Ninth International Joint Conference on Artificial Intelligence, pp. 1002-1009, Morgan Kaufmann Publisher, San Mateo, CA, USA.

Dempster, A. P. (1967). Upper and lower probabilities induced by a multivalued mapping. Annals of Mathematical Statistics, 38, 325-339.

Dubois, D., \& Prade, H. (1987). Théorie des Possibilités (2nd ed.). Paris, France: Masson.

Dubois, D., \& Prade, H. (1997). Bayesian conditioning in possibility theory. Fuzzy Sets and Systems, 92, 223-240.

Dubois, D., \& Prade, H. (1999). Editorial. Fuzzy Sets and Systems, 101(1), 1-3.

Duda, R. O., Gaschnig, J., \& Hart, P. E. (1979). Model design in the prospector consultant system for mineral exploration. In D. Michie (Ed.), Expert systems in the microelectronic age (pp. 153-167). Edinburgh, UK: Edinburgh University Press.

Eagle, A. (2005). Randomness is unpredictability. The British Journal for the Philosophy of Science, 56(4), 749-790.

Garvey, T. D., Lowrance, J. D., \& Fischler, M. A. (1981). An inference technique for integrating knowledge from disparate sources. In Proceedings of the Seventh International Joint Conference on Artificial Intelligence, pp. 319-325, Morgan Kaufmann Publisher, San Mateo, CA, USA.

Hacking, I. (1975). The emergence of probability. Cambridge, UK: Cambridge University Press.

Kolmogorov, A. N. (1963). On tables of random numbers. Sankhya. The Indian Journal of Statistics, A, 25, 369-376.

Marsaglia, G. (1995). Die Hard: A battery of tests for random number generators. http://stat.fsu.edu/ geo/diehard.html.

Martin-Löf, P. (1966). The definition of random sequences. Information and Control, 9, 602-619.

McCarthy, J., \& Hayes, P. J. (1969). Some philosophical problems from the standpoint of artificial intelligence. In B. Meltzer \& D. Michie (Eds.), Machine intelligence 4 (pp. 463-502). Edinburgh, UK: Edinburgh University Press.

Newell, A., Shaw, J. C., \& Simon, H. (1957). Empirical explorations with the logic theory machine: A case study in heuristics. In Proceedings of the Western Joint Computer Conference 15, pp. 218-239.

Newell, A., Shaw, J. C., \& Simon, H. (1958). Chess playing programs and the problem of complexity. IBM Journal of Research and Development, 2, 320-335.

Nikravesh, M. (2007). Evolution of fuzzy logic: From intelligent systems and computation to human mind. In M. Nikravesh, J. Kacprzyk, \& L. Zadeh (Eds.), Forging new frontiers: Fuzzy Pioneers I (Vol. 217, pp. 37-53). Berlin, Germany: Springer.

Nilsson, N. J. (1984). Shakey the robot. Technical Report Technical Note 323, SRI AI Center, Menlo Park, CA, USA.

Pearl, J. (1988). Probabilistic reasoning in intelligent systems. Networks of plausible inference. San Mateo, CA, USA: Morgan Kaufmann.

Popper, K. (1935). Logik der Forschung. Available as: The Logic of Scientific Discovery, London, United Kingdom: Routledge. 1999.

Shafer, G. (1976). A mathematical theory of evidence. Princeton, NJ, USA: Princeton University Press.

Shafer, G. (1978). Non additive probabilities in the work of Bernoulli and Lambert. Archive for History of Exact sciences, 19, 309-370. 
Shafer, G., \& Pearl, J. (Eds.). (1990). Uncertain reasoning. San Mateo, CA, USA: Morgan Kaufmann Publisher.

Shortliffe, E. H. (1980). Consultation systems for physicians: The role of artificial intelligence techniques. In Proceedings of the Third National Conference of the Canadian Society for Computational Studies of Intelligence, pp. 1-11.

Smets, P., \& Kennes, R. (1994). The transferable belief model. Artificial Intelligence, 66(2), 191-234.

Solo, A. M. G., \& Gupta, M. (2007). Uncertainty in computational perception and cognition. In M. Nikravesh, J. Kacprzyk, \& L. Zadeh (Eds.), Forging new frontiers: Fuzzy Pioneers I, (vol. 217, pp. 251-266). Berlin, Germany: Springer.

Sutton, R. S., \& Barto, A. G. (1998). Reinforcement learning. An introduction. Cambridge, MA, USA: MIT Press.

Tversky, A., \& Kahneman, D. (1974). Judgment under uncertainty: Heuristics and biases. Science, 185, 1124-1131.

Walley, P. (1991). Statistical reasoning with imprecise probabilities. New York, NY, USA: Chapman and Hall.

Walley, P. (1996). Measures of uncertainty in expert systems. Artificial Intelligence, 83(1), 1-58.

Walley, P. (2000). Toward a unified theory of imprecise probability. International Journal of Approximate Reasoning, 24, 125-148.

Watkins, C. J. C. H. (1989). Learning from Delayed Rewards. PhD thesis, King's College, United Kingdom: Cambridge.

Zadeh, L. A. (1965). Fuzzy sets. Information and Control, 8, 338-353.

Zadeh, L. A. (1978). Fuzzy sets as a basis for a theory of possibility. Fuzzy Sets and Systems, 1(1), 3-28.

Zadeh, L. A. (1981). Possibility theory and soft data analysis. In L. Cobb \& R. M. Thrall (Eds.), Mathematical frontiers of the social and policy sciences (pp. 69-129). Boulder, CO, USA: Westview Press.

Zadeh, L. A. (2005). Toward a generalized theory of uncertainty (GTU)—an outline. Information Sciences, 172, 1-40. 